\title{
Severity of cognitive impairment at initial diagnosis of Alzheimer's disease was the strongest predictor of survival
}

Larson EB, Shadlen MF, Wang L, et al. Survival after initial diagnosis of Alzheimer disease. Ann Intern Med 2004;140:501-9.

What is the course of disease (specifically life expectancy) after an initial diagnosis of Alzheimer's disease (AD) in community dwelling patients? Which variables are associated with survival?

\section{METHODS}

Design: inception cohort with mean follow up of 5.2 years
(range $0.2-14$ y).
Wetting: a health maintenance organisation in Seattle,
Patients: 521 patients who were $>60$ years of age $165 \%$
women, $89 \%$ white) and newly diagnosed with possible or
probable AD based on criteria of DSM-III-R (dementia) and
National Institute of Neurological and Communicative Disorders
and Stroke-Alzheimer's Disease and Related Disorders
Association (probable or possible AD).
$\begin{aligned} & \text { Prognostic factors: baseline severity of AD (Mini-Mental State } \\ & \text { Examination [MMSE] score and Dementia Rating Scale score } \\ & \text { [DRS]); psychiatric symptoms of paranoia or hallucinations; } \\ & \text { behavioural disturbances including agitation, irritability, or } \\ & \text { emotional liability; frontal lobe release signs including glabellar, } \\ & \text { snout or grasp; extrapyramidal signs including rigidity or tremor; } \\ & \text { wandering; falls; gait disorder; urinary incontinence; and } \\ & \text { depression. Variables adjusted as potential confounders included } \\ & \text { age, sex, ethnicity, education, ischaemic heart disease, } \\ & \text { congestive heart failure (CHF), diabetes, hypertension, and } \\ & \text { stroke. } \\ & \text { Outcomes: duration of survival. }\end{aligned}$

\section{MAIN RESULTS}

Median years of survival from initial diagnosis of AD was 4.2 years for men and 5.7 years for women. Proportional hazards models adjusted for age, sex, and ethnicity identified the following independent predictors of mortality: baseline MMSE score $\leqslant 17$, DRS score 5.5-17, frontal lobe release signs, gait disturbance, falls, $\mathrm{CHF}$, diabetes, and ischaemic heart disease (table).

\section{CONCLUSIONS}

Severity of cognitive impairment at initial diagnosis of Alzheimer's disease in community dwelling patients was the strongest predictor

For correspondence: $\mathrm{Dr} \mathrm{E}$ B Larson, University of Washington and the Group Health Cooperative, Seattle, WA, USA. larson.e@ghc.org

Source of funding: National Institute on Aging. of survival. Other predictors were frontal lobe release signs, gait disturbance, falls, congestive heart failure, diabetes, and ischaemic heart disease.
Comm mmentary

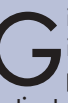
iven the aging population and the corresponding increase in the incidence of $A D$, information on the prognosis of $A D$ will help practitioners and unpaid caregivers to better meet the needs of patients with $A D$.

This well designed, prospective study by Larson et al provides estimates of survival after initial diagnosis of $A D$. Estimated survival rates were longer than those reported by the Canadian Study of Health and Aging (3.2 y for men and $3.4 \mathrm{y}$ for women). ${ }^{1}$ This difference may be attributed to the older sample in the Canadian Study of Health and Aging.

Length of survival after onset of $A D$ may be quite different from length of survival after initial diagnosis. Identifying the precise onset of $A D$ may be difficult because of the insidious nature of the disease, unpaid caregiver's lack of awareness of changes in cognition and functional abilities, and the patient's ability to compensate for impairments. Although diagnosis at the onset of disease may not alter the natural course of $A D$, it could provide opportunities to ensure that patients and their unpaid caregivers receive the most appropriate care and support throughout the course of the disease.

The importance of accurately diagnosing dementia cannot be overemphasised because different forms of dementia require different types of interventions. Patients with $A D$ primarily need support services, such as help in the home. ${ }^{2}$ Unfortunately, reduced healthcare spending in some countries has affected the availability of homemaking services. ${ }^{3}$ In addition to recommending effective strategies for managing the symptoms of $A D,{ }^{45}$ healthcare professionals should advocate for publicly funded support services required by patients with $A D$.

Dorothy Forbes, RN, PhD College of Nursing, University of Saskatchewan Saskatoon, Saskatchewan, Canada

1 Wolfson C, Wolfson DB, Asgharian M, et al. N Engl J Med 2001;344:1111-6.

2 Smale B, Dupuis S. Highlights report: preliminary results from the study on needs of caregivers of persons with Alzheimer Disease or a related dementia and community support services in Ontario, June 2002. Murray Alzheimer Research and Education Program, University of Waterloo,

Waterloo, Ontario. http://marep.uwaterloo.ca/projects.html

3 Forbes DA, Stewart N, Morgan D, et al. Can J Nurs Res 2003;35:14-36.

4 Forbes DA. Can J Nurs Res 1998;30:67-86.

5 Peacock SC, Forbes DA. Can J Nurs Res 2003;35:88-107.

Prognostic factors for mortality in community dwelling patients with newly diagnosed Alzheimer's disease

\begin{tabular}{lll}
\hline Prognostic factor & Median years of survivalt & Hazard ratio $(95 \%$ Cl)‡ \\
\hline MMSE score $\leqslant 17$ & 3.6 & $2.58(1.88$ to 3.53$)$ \\
DRS score 5.5-17 & 3.2 & $2.15(1.62$ to 2.84$)$ \\
Frontal lobe release signs & 4.6 & $1.26(1.03$ to 1.54$)$ \\
Gait disturbance & 3.5 & $1.50(1.20$ to 1.87$)$ \\
History of falls & 4.3 & $1.30(1.05$ to 1.60$)$ \\
Ischaemic heart disease & 4.1 & $1.30(1.05$ to 1.61$)$ \\
Congestive heart failure & 3.0 & $1.48(1.10$ to 1.99$)$ \\
Diabetes & 3.8 & $1.87(1.34$ to 2.61$)$ \\
\hline
\end{tabular}

${ }^{*}$ MMSE $=$ Mini Mental State Examination; DRS = Dementia Rating Scale; other abbreviations defined in glossary

†Time until death when $50 \%$ of patients died.

fIndependent prognostic factors based on proportional hazards model adjusted for age at diagnosis, sex, and ethnicity. 